\title{
Cutaneous Crust
}

National Cancer Institute

\section{Source}

National Cancer Institute. Cutaneous Crust. NCI Thesaurus. Code C120873.

A hard covering or layer of solid matter formed by dried bodily exudate or secretion. 